\title{
Relação entre solos e unidades da paisagem no ecossistema Pantanal
}

\author{
Evaldo Luis Cardoso(1), Sandra Aparecida Santos(1), Catia Urbanetz(1), \\ Amaury de Carvalho Filho(2), Uebi Jorge Naime ${ }^{(2)}$, Marx Leandro Naves Silva ${ }^{(3)}$ e Nilton Curi ${ }^{(3)}$
}

\begin{abstract}
(1)Embrapa Pantanal, Rua 21 de Setembro, no 1.880, Nossa Senhora de Fátima, CEP 79320-900 Corumbá, MS, Brasil. E-mail: evaldo. cardoso@embrapa.br, sandra.santos@embrapa.br, catia.urbanetz@embrapa.br (2)Embrapa Solos, Rua Jardim Botânico, no 1.024, Jardim Botânico, CEP 22460-000 Rio de Janeiro, RJ, Brasil. E-mail: amaury.carvalho@embrapa.br, uebijn@gmail.com (3)Universidade Federal de Lavras, Departamento de Ciência do Solo, Caixa Postal 3037, CEP 37200-000 Lavras, MG, Brasil. E-mail: marx@dcs.ufla.br, niltcuri@dcs.ufla.br
\end{abstract}

Resumo - O objetivo deste trabalho foi avaliar as variações nos solos e em seus atributos químicos sob diferentes unidades da paisagem no ecossistema Pantanal, na sub-região da Nhecolândia, no Mato Grosso do Sul. As unidades da paisagem avaliadas incluíram: FS, floresta semidecídua; CE, cerradão; CC, cerrado/ campo cerrado; CLE, campo limpo com Elionurus muticus; CLA, campo limpo com Axonopus purpusii e Andropogon spp.; BB, bordas de baías; e VB, vazantes/baixadas. Perfis de solos representativos de cada unidade da paisagem foram descritos morfologicamente, e os atributos químicos foram determinados nas profundidades de $0,00-0,05,0,05-0,10,0,10-0,15$ e $0,15-0,20 \mathrm{~m}$. Os solos apresentaram textura arenosa, baixo teor de matéria orgânica, e baixa capacidade de troca catiônica e aniônica. A principal diferenciação entre os solos avaliados foi a fertilidade natural, a qual foi mais elevada sob FS, especialmente nas camadas mais superficiais e nos horizontes abaixo de $2 \mathrm{~m}$ de profundidade. A qualidade química do solo é capaz de discriminar as unidades da paisagem em três diferentes grupos: 1, FS; 2, CE; e 3, CC, CLE, CLA, BB e VB.

Termos para indexação: área úmida, composição florística, conservação ambiental, funcionamento da paisagem, Pantanal da Nhecolândia, sistemas naturais.

\section{Relationship between soils and landscape units in the Pantanal ecosystem, in Brazil}

\begin{abstract}
The objective of this work was to evaluate the changes in soils and their chemical attributes under different landscape units in the Pantanal ecosystem, in the Nhecolândia subregion, in the state of Mato Grosso do Sul, Brazil. The evaluated landscape units included: SF, semideciduous forest; CE, "cerradão"; CC, "cerrado"/“campo cerrado"; TPE, tropical open grassland with Elionurus muticus; TPA, tropical open grassland with Axonopus purpusii and Andropogon spp.; EP, edge of ponds; and TPL, temporary channels/ lowlands. Soil profiles representative of each landscape unit were morphologically described, and chemical attributes were determined in the depths of $0.00-0.05,0.05-0.10,0.10-0.15$, and $0.15-0.20 \mathrm{~m}$. The soils showed sandy texture, low soil organic matter content, and low cation and anion exchange capacity. The main difference between the evaluated soils was natural fertility, which was higher in the SF, particularly at the surface layers and at the horizons below 2-m depth. Soil chemical quality is able to discriminate the landscape units into three different groups: 1, SF; 2, CE; and 3, CC, TPE, TPA, EP, and TPL.

Index terms: wetland, floristic composition, environmental conservation, landscape funcioning, Pantanal of Nhecolândia, natural systems.
\end{abstract}

\section{Introdução}

A composição e a estrutura da paisagem do ecossistema Pantanal são bastante diversificadas, sendo formadas por um mosaico de distintas unidades da paisagem, fitofisionomias, solos e gradientes de inundação. Nesse ecossistema de rica biodiversidade, encontram-se: florestas como as matas semidecíduas e o cerradão, localizadas em áreas conhecidas regionalmente como "cordilheiras"; florestas ripárias; formações monodominantes, como carandazais, cambarazais e paratudais; e matas secas ou florestas decíduas. Também ocorrem formações savânicas, como cerrado sensu stricto, campo cerrado e campo sujo; formações campestres, com alagamentos eventuais, como campo limpo, campos inundáveis e vazantes; e ambientes aquáticos permanentes ou 
temporários, com água salobra ou doce (Coutinho, 2006).

Os solos na paisagem podem ser vistos, segundo Sommer (2006), sob a ótica da variabilidade espaçotemporal de seus atributos e sob a perspectiva de processos dinâmicos, como, por exemplo, o de transporte de água, solutos e sedimentos. Conforme o autor, para uma compreensão mais abrangente do solo na paisagem, o grande desafio é entender a interação entre ambos. No entanto, a criação de bancos de dados de solos do Brasil ainda é incipiente e sabe-se pouco sobre a relação entre a distribuição das formações vegetais nativas e a fertilidade natural dos solos (Skorupa et al., 2012). As variações nos atributos químicos do solo, aliadas ao gradiente topográfico, tendem a exercer forte influência na composição florística e na abundância de espécies nas unidades da paisagem no Pantanal. Carvalho Filho et al. (2000) apontam que a ocorrência predominante da palmeira acuri (Attalea phalerata Mart. ex Spreng.) nas áreas florestadas desse ecossistema denota, provavelmente, uma melhor condição química do solo, representada por maiores valores de soma de bases e teores de fósforo disponível. Nos ambientes mais fechados e nos arredores das salinas, a maior riqueza de nutrientes favorece a presença do assa-peixe (Vernonia sp.), espécie praticamente ausente nas demais áreas, e também de aglomerados de carandá (Copernicia alba Morong ex Morong \& Britton), palmeira relacionada a teores mais elevados de sódio no solo. Já a ocorrência de muitos indivíduos de Qualea grandiflora Mart. em dois cerradões da Nhecolândia, no Mato Grosso do Sul, foi associada à maior disponibilidade de fósforo (Salis et al., 2006).

Estudos mais recentes sobre os solos do Pantanal têm buscado compreender sua gênese quando adjacentes às lagoas salinas (Barbiero et al., 2008; Furquim et al., 2008, 2010), bem como os processos de migração, dispersão e concentração de elementos químicos no seu perfil, por meio da análise geoquímica dos solos de diferentes ambientes (Coringa et al., 2014). Entretanto, ainda são pouco conhecidas as variações nos atributos dos solos associadas a unidades da paisagem como florestas, cerradões, cerrados, vazantes e bordas de baías, as quais têm destacada importância nos sistemas extensivos de criação de gado de corte no Pantanal. O manejo sustentável dos recursos naturais de sistemas complexos, como o Pantanal, deve ter como premissas imprescindíveis a compreensão dos processos ecológicos responsáveis pela sua produtividade e sua biodiversidade, e, fundamentalmente, o conhecimento sobre como os componentes bióticos e abióticos se inter-relacionam.

O objetivo deste trabalho foi avaliar as variações nos solos e em seus atributos químicos sob diferentes unidades da paisagem no ecossistema Pantanal, na sub-região da Nhecolândia, no Mato Grosso do Sul.

\section{Material e Métodos}

O trabalho foi conduzido na área experimental da Embrapa Pantanal, cuja paisagem é representativa da parte mais alta da sub-região da Nhecolândia, que apresenta área aproximada de $26.000 \mathrm{~km}^{2}$ e situa-se à margem esquerda do rio Taquari. Essa é uma das 11 sub-regiões do Pantanal, delimitadas de acordo com aspectos relativos à inundação, ao relevo, ao solo e à vegetação (Silva \& Abdon, 1998).

As unidades da paisagem estudadas localizamse entre as latitudes $18^{\circ} 59^{\prime} 06^{\prime \prime}$ e $19^{\circ} 00^{\prime} 06^{\prime \prime}$ sul, e as longitudes 56 39'40" e 56 40’40" oeste (Santos et al., 2002). Nas áreas mais altas e livres de inundação, as unidades da paisagem foram especificadas da seguinte forma: FS, floresta semidecídua, caracterizada pela predominância de $A$. phalerata no seu interior e pela diversidade de espécies nas bordas, com destaque para Arrabidaea sp., Cecropia pachystachya Trécul e Smilax fluminensis Steud.; e CE, cerradão, vegetação xeromorfa sobre "cordilheiras" arenosas, cuja composição florística é bastante heterogênea, com destaque para Magonia pubescens A.St.-Hil., Handroanthus impetiginosus (Mart. ex DC.) Mattos, Anadenanthera colubrina (Vell.) Brenan, Astronium fraxinifolium Schott, Sterculia apetala (Jacq.) H. Karst. e A. phalerata. Nas áreas de cotas intermediárias, sujeitas à inundação ocasional em grandes cheias, as unidades da paisagem foram especificadas como: $\mathrm{CC}$, cerrado/campo cerrado, cujo estrato herbáceo, dominado principalmente por Mesosetum chaseae Luces, encontra-se sob plantas lenhosas esparsas, como Byrsonima cydoniifolia A. Juss., Curatella americana L. e Annona dioica A.St.-Hil.; e CLE, campo limpo com predominância de Elionurus muticus (Spreng.) Kuntze. Nas áreas mais baixas, sujeitas à inundação sazonal, as especificações foram: CLA, campo limpo com predominância de Axonopus 
purpusii (Mez) Chase e Andropogon spp., situado na paisagem em posição um pouco mais rebaixada que o CLE; BB, bordas de baías permanentes, caracterizadas pela predominância de Hymenachne amplexicaulis (Rudge) Nees, Leersia hexandra Sw., Panicum laxum Sw., ciperáceas e outras macrófitas; e VB, vazantes/ baixadas, em que as vazantes são vias de drenagem não seccionadas, e as baixadas são pequenos desníveis do relevo, marcadas pela predominância de gramíneas hidrófilas como P. laxum, Setaria geniculata (Lam.) P.Beauv. e ciperáceas.

A partir de três transectos de $100 \mathrm{~m}$, demarcados em cada unidade da paisagem, foram coletadas subamostras de solo, a cada $20 \mathrm{~m}$, nas profundidades de $0,00-0,05,0,05-0,10,0,10-0,15$ e $0,15-0,20 \mathrm{~m}$, com três repetições por unidade da paisagem. As amostras de solo foram secas ao ar, passadas em peneiras de malha de $2 \mathrm{~mm}$ de diâmetro e submetidas às seguintes análises químicas, de acordo com Claessen (1997): $\mathrm{pH}$ em água (1:2,5); $\mathrm{Ca}^{2+}, \mathrm{Mg}^{2+}$ e $\mathrm{Al}^{3+}$ trocáveis, extraídos com solução de $\mathrm{KCl} 1 \mathrm{~mol} \mathrm{~L}^{-1}$ e determinados por espectrofotometria de absorção atômica; $\mathrm{P}$ disponível, e $\mathrm{K}^{+}$e $\mathrm{Na}^{+}$trocáveis, extraídos com solução ácida (Mehlich-1), com o P quantificado por colorimetria, e $\mathrm{K}^{+}$e $\mathrm{Na}^{+}$por fotometria de chama; $\mathrm{H}+\mathrm{Al}$, extraídos com solução de acetato de cálcio tamponada a pH 7,0; e matéria orgânica do solo (MOS), determinada por oxidação com dicromato de potássio. A partir dos resultados, foram calculadas a soma de bases (SB), a capacidade de troca de cátions efetiva $\left(\mathrm{CTC}_{\mathrm{t}}\right)$, e a percentagem de saturação por bases (V) e por alumínio.

Os perfis de solos, representativos de cada unidade da paisagem, foram descritos morfologicamente em trincheiras abertas para este fim, como em Santos et al. (2005), e classificados pelos critérios estabelecidos por Santos et al. (2013). As amostras coletadas em cada horizonte foram submetidas a análises físicas e químicas de acordo com Claessen (1997). Os horizontes estudados abaixo de $2 \mathrm{~m}$ de profundidade foram amostrados da mesma forma que os superficiais, para acessar a expressão da atividade biológica e dos processos pedogenéticos. Com uso de nível topográfico e tendo-se como referência o fundo central da maior baía presente na área, foram determinadas pelo menos três cotas topográficas em cada unidade da paisagem.

Os atributos químicos do solo foram submetidos à análise de variância, com uso do programa Sisvar (Universidade Federal de Lavras, Lavras, MG), tendo- se adotado o delineamento experimental inteiramente casualizado, com três repetições. As comparações múltiplas de médias foram realizadas com o teste de Tukey, a 5\% de probabilidade. Ainda foram realizadas análises multivariadas, de agrupamento e ordenação, para identificação de possíveis diferenças nos atributos do solo de acordo com a estrutura da vegetação. Os dendrogramas foram obtdos com os métodos de ligação de Ward (variância mínima) simples, completa e média de grupo (UPGMA), bem como com o índice de distância euclidiana simples. O cálculo do índice de correlação cofenética foi realizado com todos os dendrogramas, e a análise de componentes principais (PCA) foi feita com uso da matriz de correlação (Jackson, 1991). Os dados foram transformados para padronizar as diferentes unidades de cada variável, por meio da transformação por estandardização, isto é, da divisão pela média.

\section{Resultados e Discussão}

Em geral, os solos das unidades da paisagem caracterizam-se pela dominância de cores pálidas, com matiz 10YR, com ocorrência comum de horizontes com intenso mosqueamento, e de textura arenosa em toda a extensão dos perfis (Tabela 1). De acordo com o Sistema Brasileiro de Classificação de Solos (Santos et al., 2013), enquadram-se, em segundo nível categórico, na classe dos Neossolos Quartzarênicos; e em função da menor ou maior influência de condições de hidromorfismo, diferenciam-se, em nível subsequente, como Neossolos Quartzarênicos órticos e Neossolos Quartzarênicos hidromórficos, estes últimos situados nas posições mais rebaixadas, onde o lençol freático encontra-se mais próximo da superfície (Figura 1). Também Barbiero et al. (2008) verificaram, ao longo das cordilheiras, até as vazantes, a dominância de Neossolos Quartzarênicos, com teores de argila inferiores a $35 \mathrm{~g} \mathrm{~kg}^{-1} \mathrm{e} \mathrm{pH}$ de neutro a extremamente ácido.

As áreas mais elevadas, livres de inundação, representadas por FS e CE, apresentaram cotas médias de 2,53 e 2,30 m, respectivamente, e Neossolos Quartzarênicos órticos típicos. Na FS, o horizonte A é relativamente espesso e de cor escura, mas os teores de $\mathrm{CO}$ são bastante reduzidos, o que leva ao reconhecimento do horizonte A moderado. Já no CE, 
Tabela 1. Caracterização dos perfis dos solos das diferentes unidades da paisagem do Pantanal, na sub-região da Nhecolândia, no Mato Grosso do Sul.

\begin{tabular}{|c|c|c|c|c|c|c|c|c|c|c|c|c|c|c|}
\hline \multirow[t]{2}{*}{ Horizonte } & \multirow{2}{*}{$\begin{array}{l}\text { Profundidade } \\
\text { (m) }\end{array}$} & \multicolumn{2}{|c|}{$\operatorname{Cor}^{(1)}$} & \multicolumn{4}{|c|}{ Granulometria $^{(2)}\left(\mathrm{g} \mathrm{kg}^{-1}\right)$} & \multirow[t]{2}{*}{$\mathrm{CO}$} & \multirow{2}{*}{$\begin{array}{l}\mathrm{pH} \\
\mathrm{H}_{2} \mathrm{O}\end{array}$} & \multirow{2}{*}{$\begin{array}{c}\mathrm{SB} \\
\left(\mathrm{cmol}_{\mathrm{c}} \mathrm{dm}^{-3}\right)\end{array}$} & \multirow{2}{*}{$\begin{array}{l}\mathrm{V} \\
(\%)\end{array}$} & \multirow{2}{*}{$\begin{array}{l}\text { Saturação } \\
\text { por } \mathrm{Na}^{+}(\%)\end{array}$} & \multirow{2}{*}{$\begin{array}{c}\mathrm{P} \\
\left(\mathrm{mg} \mathrm{kg}^{-1}\right)\end{array}$} & \multirow[t]{2}{*}{ Classe textural } \\
\hline & & Seca & Úmida & $\mathrm{Ag}$ & Af & Silte & Argila & & & & & & & \\
\hline & \multicolumn{14}{|c|}{ Floresta semidecídua, Neossolo Quartzarênico órtico típico, A moderado } \\
\hline A1 & $0,00-0,12$ & 10 YR $5 / 3$ & $10 \mathrm{YR} 3 / 3$ & 280 & 600 & 80 & 40 & 7,6 & 5,4 & 2,0 & 53 & $<1$ & 14 & Areia \\
\hline A2 & $-0,40$ & 10YR $5 / 3$ & $10 \mathrm{YR} 3 / 3$ & 300 & 620 & 40 & 40 & 3,7 & 6,0 & 1,3 & 46 & $<1$ & 16 & Areia \\
\hline A3 & $-0,55$ & 10YR 5/4 & 10YR 3/4 & 320 & 580 & 60 & 40 & 1,8 & 6,3 & 1,0 & 56 & $<1$ & 10 & Areia \\
\hline $\mathrm{AC}$ & $-0,96$ & - & $10 \mathrm{YR} 4 / 4$ & 240 & 640 & 80 & 40 & 1,2 & 6,6 & 0,8 & 73 & $<1$ & 9 & Areia \\
\hline $\mathrm{CA}$ & $-1,34$ & - & $10 \mathrm{YR} 4 / 4 \mathrm{M}$ & 310 & 600 & 40 & 50 & 0,9 & 6,6 & 0,5 & 62 & 1 & 4 & Areia \\
\hline $\mathrm{C} 1$ & $-1,74$ & - & $10 Y R 6 / 4 \mathrm{~V}$ & 310 & 560 & 80 & 50 & 0,2 & 6,7 & 0,4 & 57 & 1 & 3 & Areia franca \\
\hline $\mathrm{C} 2$ & $-2,14$ & - & 10 YR $6 / 4$ & 330 & 540 & 80 & 50 & 0,6 & 7,6 & 0,8 & 80 & 1 & 1 & Areia franca \\
\hline $\mathrm{Cn} 1$ & $-2,67$ & - & $10 \mathrm{YR} 6 / 5 \mathrm{M}$ & 270 & 640 & 40 & 50 & 0,6 & 9,6 & 1,2 & 100 & 11 & 33 & Areia \\
\hline $\mathrm{Cn} 2$ & $-2,87$ & - & $10 \mathrm{YR} 5 / 3 \mathrm{M}$ & 180 & 620 & 140 & 60 & 0,6 & 10,0 & 2,9 & 100 & 17 & 9 & Areia franca \\
\hline $\mathrm{Cn} 3$ & $-2,98$ & - & $10 \mathrm{YR} 6 / 3 \mathrm{~V}$ & 160 & 700 & 80 & 60 & 0,7 & 9,9 & 2,8 & 100 & 18 & 20 & Areia franca \\
\hline \multirow[t]{2}{*}{$\mathrm{Cn} 4$} & $-3,27$ & - & $10 \mathrm{YR} 5 / 3 \mathrm{~V}$ & 170 & 620 & 150 & 60 & 0,7 & 9,6 & 3,1 & 100 & 18 & 15 & Areia franca \\
\hline & \multicolumn{14}{|c|}{ Cerradão, Neossolo Quartzarênico órtico típico, A fraco } \\
\hline A1 & $0,00-0,09$ & - & $10 Y R 4 / 3$ & 370 & 570 & 10 & 50 & 2,9 & 4,5 & 0,4 & 14 & $<1$ & 8 & Areia \\
\hline $\mathrm{A} 2$ & $-0,37$ & - & $10 Y R 4 / 4$ & 370 & 570 & 10 & 50 & 2,0 & 4,6 & 0,3 & 17 & $<1$ & 1 & Areia \\
\hline $\mathrm{CA}$ & $-0,79$ & - & $10 \mathrm{YR} 5 / 3$ & 350 & 590 & 10 & 50 & 1,3 & 4,7 & 0,2 & 13 & $<1$ & 1 & Areia \\
\hline $\mathrm{C} 1$ & $-1,22$ & - & $10 \mathrm{YR} 7 / 3$ & 330 & 610 & 10 & 50 & 0,5 & 5,3 & 0,1 & 7 & $<1$ & 2 & Areia \\
\hline $\mathrm{C} 2$ & $-1,34$ & - & 10YR 7/4 & 320 & 620 & 10 & 50 & 0,5 & 5,2 & 0,1 & 8 & $<1$ & 5 & Areia \\
\hline $\mathrm{C} 3$ & $-1,55$ & - & $10 \mathrm{YR} 6 / 6 \mathrm{~V}$ & 320 & 620 & 10 & 50 & 0,5 & 5,1 & 0,1 & 7 & $<1$ & 4 & Areia \\
\hline $\mathrm{C} 4$ & $-1,70$ & - & 10 YR $6 / 4 \mathrm{~V}$ & 330 & 610 & 10 & 50 & 0,3 & 5,3 & 0,1 & 10 & 1 & 2 & Areia \\
\hline & & & & Cerr & camp & errado, & eossolo & uartzo & nico & tico típico, A f & & & & \\
\hline A & $0,00-0,14$ & 10 YR $6 / 3$ & $10 \mathrm{YR} 4 / 3$ & 240 & 680 & 40 & 40 & 4,3 & 4,8 & 0,4 & 18 & $<1$ & 3 & Areia \\
\hline $\mathrm{AC}$ & $-0,40$ & $10 Y R 7 / 3$ & $10 Y R 5 / 3$ & 390 & 480 & 90 & 40 & 1,3 & 5,1 & 0,1 & 12 & 1 & 1 & Areia franca \\
\hline CA & $-0,77$ & - & $10 Y R 5 / 3$ & 320 & 600 & 40 & 40 & 1,3 & 5,3 & 0,2 & 29 & 1 & 1 & Areia \\
\hline $\mathrm{C} 1$ & $-1,02$ & - & $10 Y R 6 / 3$ & 340 & 610 & 10 & 40 & 0,6 & 5,0 & 0,2 & 29 & 1 & 1 & Areia \\
\hline $\mathrm{C} 2$ & $-1,87$ & - & $10 Y R 7 / 3$ & 360 & 500 & 90 & 50 & 0,2 & 5,4 & 0,1 & 33 & 3 & 1 & Areia franca \\
\hline $\mathrm{Cg}$ & $-1,97$ & - & 10 YR $6 / 2$ & 360 & 560 & 30 & 50 & 0,3 & 4,9 & 0,1 & 11 & 1 & 3 & Areia \\
\hline & & & & & LE, $N$ & solo Q & rtzarêni & órtic & típico & A moderado & & & & \\
\hline A & $0,00-0,07$ & 10 YR $5 / 2$ & $10 \mathrm{YR} 4 / 3$ & 240 & 660 & 50 & 50 & 3,7 & 5,4 & 0,3 & 17 & $<1$ & 4 & Areia \\
\hline $\mathrm{AC}$ & $-0,15$ & 10 YR $6 / 3$ & $10 \mathrm{YR} 4 / 3$ & 210 & 700 & 40 & 50 & 2,9 & 5,2 & 0,2 & 12 & $<1$ & 1 & Areia \\
\hline $\mathrm{CA}$ & $-0,31$ & - & $10 Y R 4 / 4$ & 230 & 620 & 100 & 50 & 1,7 & 5,3 & 0,1 & 8 & $<1$ & 1 & Areia franca \\
\hline $\mathrm{C} 1$ & $-0,54$ & - & $10 Y R 5 / 4$ & 230 & 660 & 60 & 50 & 1,3 & 5,2 & 0,1 & 9 & $<1$ & 1 & Areia \\
\hline $\mathrm{C} 2$ & $-0,78$ & - & $10 \mathrm{YR} 6 / 3 \mathrm{M}$ & 220 & 680 & 50 & 50 & 1,0 & 5,1 & 0,2 & 20 & 1 & 1 & Areia \\
\hline $\mathrm{C} 3$ & $-1,19$ & - & $10 \mathrm{YR} 6 / 6 \mathrm{M}$ & 210 & 720 & 20 & 50 & 0,5 & 5,1 & 0,1 & 12 & 1 & 1 & Areia \\
\hline $\mathrm{C} 4$ & $-1,52$ & - & $10 \mathrm{YR} 6 / 4 \mathrm{M}$ & 220 & 680 & 50 & 50 & 0,6 & 5,4 & 0,1 & 12 & 1 & 1 & Areia \\
\hline & & & & & Neos & o Quar & rênico & drome & ico típ & co, A moderad & & & & \\
\hline A & $0,00-0,06$ & $10 \mathrm{YR} 6 / 2$ & $10 \mathrm{YR} 3 / 3$ & 210 & 700 & 30 & 60 & 7,6 & 5,2 & 0,5 & 18 & $<1$ & 1 & Areia \\
\hline $\mathrm{AC}$ & $-0,22$ & $10 \mathrm{YR} 6 / 3$ & $10 \mathrm{YR} 4 / 3$ & 230 & 690 & 20 & 60 & 2,3 & 5,2 & 0,4 & 19 & $<1$ & 1 & Areia \\
\hline $\mathrm{CA}$ & $-0,38$ & - & 10YR 5/4 & 240 & 690 & 10 & 60 & 1,0 & 5,8 & 0,5 & 33 & 2 & 1 & Areia \\
\hline $\mathrm{C}$ & $-0,64$ & - & 10YR 6/4 & 270 & 600 & 80 & 50 & 0,4 & 6,1 & 0,3 & 37 & 4 & 1 & Areia franca \\
\hline $\mathrm{Cn}$ & $-0,95$ & - & $10 Y R 6 / 4$ & 250 & 630 & 70 & 50 & 0,3 & 7,0 & 0,4 & 44 & 6 & 1 & Areia \\
\hline$C^{\prime}$ & $-1,21$ & - & $10 Y R 6 / 4$ & 260 & 640 & 50 & 50 & 0,4 & 7,0 & 0,7 & 50 & 4 & 1 & Areia \\
\hline C'n & $-1,32$ & - & $10 Y R 6 / 4$ & 250 & 620 & 80 & 50 & 0,3 & 6,7 & 0,4 & 57 & 9 & 2 & Areia franca \\
\hline $\mathrm{Cg}$ & $-1,52$ & - & $10 \mathrm{YR} 5 / 2$ & 260 & 670 & 20 & 50 & 0,5 & 6,2 & 0,7 & 50 & 4 & 1 & Areia \\
\hline & & & & 3ordas & baías, & ossolo & uartzarê & co hic & omórfi & o típico, A moc & erado & & & \\
\hline A & $0,00-0,3$ & - & $10 \mathrm{YR} 2 / 2$ & 180 & 630 & 140 & 50 & 42,6 & 5,6 & 2,5 & 28 & 2 & 4 & Areia franca \\
\hline $\mathrm{AC}$ & $0,05-15$ & - & $10 \mathrm{YR} 3 / 2$ & 260 & 620 & 70 & 50 & 3,9 & 5,3 & 0,4 & 18 & $<1$ & 1 & Areia \\
\hline CA & $-0,26$ & - & $10 \mathrm{YR} 5 / 3 \mathrm{M}$ & 240 & 640 & 70 & 50 & 1,5 & 5,2 & 0,3 & 20 & $<1$ & 1 & Areia \\
\hline $\mathrm{C}$ & $-0,43$ & - & $10 Y R 6 / 3$ & 250 & 680 & 20 & 50 & 0,8 & 5,5 & 0,3 & 27 & $<1$ & 1 & Areia \\
\hline $\mathrm{Cg}$ & $-0,79$ & - & 10YR $6 / 2$ & 260 & 640 & 50 & 50 & 0,6 & 5,4 & 0,2 & 22 & 1 & 1 & Areia \\
\hline & & & & antes/ & adas, & ossolo & artzar & co hid & mórfi & espódico, A & oderac & & & \\
\hline A1 & $0,00-0,04$ & 10 YR $6 / 3$ & $10 \mathrm{YR} 4 / 3$ & 250 & 670 & 40 & 40 & 8,2 & 6,1 & 0,8 & 32 & 5 & 2 & Areia \\
\hline A2 & $-0,16$ & $10 \mathrm{YR} 6 / 3$ & $10 \mathrm{YR} 4 / 4$ & 280 & 660 & 20 & 40 & 2,5 & 6,0 & 0,4 & 33 & 4 & 1 & Areia \\
\hline $\mathrm{AC}$ & $-0,22$ & - & $10 \mathrm{YR} 5 / 4 \mathrm{M}$ & 280 & 660 & 20 & 40 & 1,7 & 6,2 & 0,3 & 50 & 8 & 1 & Areia \\
\hline
\end{tabular}

Continua... 
Tabela 1. Continuação.

\begin{tabular}{|c|c|c|c|c|c|c|c|c|c|c|c|c|c|c|}
\hline \multirow[t]{2}{*}{ Horizonte } & \multirow{2}{*}{$\begin{array}{l}\text { Profundidade } \\
\text { (m) }\end{array}$} & \multicolumn{2}{|c|}{$\operatorname{Cor}^{(1)}$} & \multicolumn{4}{|c|}{ Granulometria $^{(2)}\left(\mathrm{g} \mathrm{kg}^{-1}\right)$} & \multirow[t]{2}{*}{$\mathrm{CO}$} & \multirow{2}{*}{$\begin{array}{c}\mathrm{pH} \\
\mathrm{H}_{2} \mathrm{O}\end{array}$} & \multirow{2}{*}{$\begin{array}{c}\mathrm{SB} \\
\left(\mathrm{cmol}_{\mathrm{c}} \mathrm{dm}^{-3}\right)\end{array}$} & \multirow{2}{*}{$\begin{array}{l}\mathrm{V} \\
(\%)\end{array}$} & \multirow{2}{*}{$\begin{array}{c}\text { Saturação } \\
\text { por } \mathrm{Na}^{+}(\%)\end{array}$} & \multirow{2}{*}{$\begin{array}{c}\mathrm{P} \\
\left(\mathrm{mg} \mathrm{kg}^{-1}\right)\end{array}$} & \multirow[t]{2}{*}{ Classe textura } \\
\hline & & Seca & Úmida & $\mathrm{Ag}$ & Af & Silte & Argila & & & & & & & \\
\hline$\overline{\mathrm{C} 1}$ & $-0,41$ & - & 10YR $6 / 6$ & 280 & 660 & 20 & 40 & 1,1 & 6,3 & 0,3 & 60 & 10 & 1 & Areia \\
\hline $\mathrm{C} 2$ & $-0,63$ & - & 10 YR $7 / 4$ & 340 & 520 & 90 & 50 & 0,7 & 6,7 & 0,3 & 60 & 8 & 1 & Areia franca \\
\hline $\mathrm{C} 3$ & $-0,95$ & - & 10 YR $6 / 6 \mathrm{~V}$ & 330 & 560 & 50 & 60 & 0,7 & 6,4 & 1,0 & 67 & 7 & 1 & Areia \\
\hline $\mathrm{C} 4$ & $-1,23$ & - & $10 \mathrm{YR} 7 / 8 \mathrm{~V}$ & 320 & 560 & 60 & 60 & 1,0 & 6,3 & 1,9 & 86 & 4 & 1 & Areia franca \\
\hline $\mathrm{Cg}$ & $-1,54$ & - & $2,5 Y 5 / 2$ & 320 & 540 & 50 & 90 & 1,1 & 5,9 & 3,0 & 75 & 3 & 2 & Areia franca \\
\hline
\end{tabular}

${ }^{(1)}$ Cor dominante: M, com mosqueamento abundante; e V, com coloração variegada. ${ }^{(2)} \mathrm{Ag}$, areia grossa; e Af, areia fina. CO, carbono orgânico; SB, soma de bases trocáveis; V, saturação por bases; P, fósforo disponível; CLE, campo limpo com predominância de Elionurus muticus; e CLA, campo limpo com predominância de Axonopus purpusii e Andropogon spp.

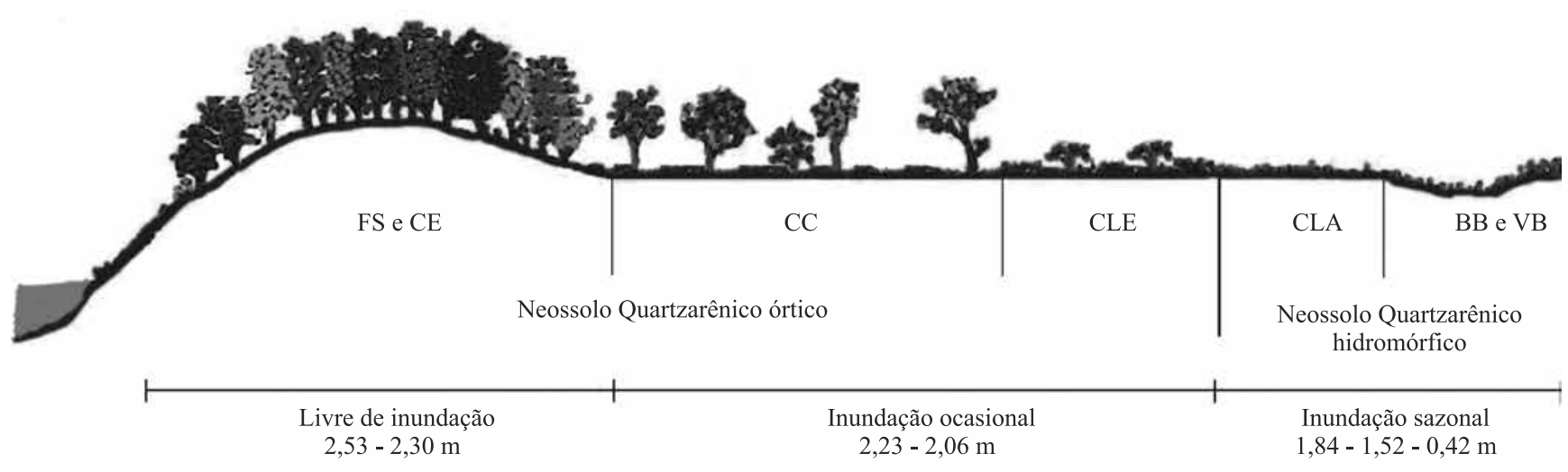

Figura 1. Solo predominante, regime de inundação e cotas médias de diferentes unidades da paisagem do Pantanal, na subregião da Nhecolândia, no Mato Grosso do Sul. FS, floresta semidecídua; CE, cerradão; CC, cerrado/campo cerrado; CLE, campo limpo com predominância de Elionurus muticus; CLA, campo limpo com predominância de Axonopus purpusii $\mathrm{e}$ Andropogon spp.; BB, bordas de baías; e VB, vazantes/baixadas.

Fonte: adaptado de Cunha (1980).

as cores claras e o conteúdo de $\mathrm{CO}$, ainda mais baixo, caracterizam o horizonte A fraco.

A principal diferenciação entre os solos dessas unidades da paisagem, porém, decorre das condições de fertilidade natural, a qual é bem mais elevada na FS, especialmente nos horizontes abaixo de 2 $\mathrm{m}$ de profundidade, inclusive com concentração de $\mathrm{Na}^{+}$relativamente alta e teores de $\mathrm{P}$ disponível mais elevados (Tabela 1).

As áreas sujeitas à inundação ocasional, representadas por CLE e CC, apresentaram cotas médias de 2,06 e 2,23 m, respectivamente, e Neossolos Quartzarênicos órticos típicos, com pouca diferenciação de horizontes e predomínio de cores claras, inclusive na camada superficial, que em associação com os teores de $\mathrm{CO}$ muito baixos, distinguem o horizonte A fraco. É marcante o processo de lixiviação no perfil do solo sob CLE e CC, evidenciado por teores de nutrientes muito reduzidos. Esse processo é determinado principalmente pelas frequentes oscilações do lençol freático, durante quase seis meses por ano. Estudos sobre solos adjacentes às lagoas salinas na região indicam a participação efetiva do processo de lixiviação na eliminação gradual dos cátions bivalentes $(\mathrm{Ca} 2+, \mathrm{Mg} 2+)$ do complexo de troca e sua paulatina substituição por $\mathrm{Na}+$ (Furquim et al., 2008, 2010).

As áreas mais rebaixadas e sujeitas à inundação sazonal, representadas por $\mathrm{VB}, \mathrm{BB}$ e CLA, apresentaram cotas médias de 0,42, 1,52 e 1,84 $\mathrm{m}$, respectivamente, e Neossolos Quartzarênicos hidromórficos, caracterizados pela presença do lençol freático a menos de $1,5 \mathrm{~m}$ da superfície do solo durante a época seca ou pela ocorrência de horizonte glei até 
essa profundidade, apesar de submetidos a condições de maior umidade, o conteúdo de CO desses solos também é bastante baixo, à exceção de camadas muito estreitas, à superfície; além disso, são inexpressivos os teores de nutrientes.

Sob condições naturais ácidas, os atributos químicos do solo das diferentes unidades da paisagem refletem sua textura arenosa, seu baixo teor de matéria orgânica, e sua baixa capacidade de retenção de cátions e ânions (Tabela 2). Em geral, notadamente nas profundidades de $0,0-0,05$ e $0,05-0,10 \mathrm{~m}$, constatou-se melhoria relativa na qualidade química do solo, nas áreas livres de inundação e caracterizadas por vegetação arbórea de maior porte, representadas, sobretudo, pela FS. A acidez ativa $(\mathrm{pH})$ do solo variou significativamente em todas as profundidades, com valores de 6,2 a 6,6 na FS, e de 4,8 a 5,6 nas demais unidades, valores que representam, respectivamente, acidez fraca e média à elevada (Alvarez V. et al., 1999). A maior acidez, principalmente nas unidades da paisagem $\mathrm{VB}$ e BB, pode estar relacionada à presença de MOS não decomponível, depositada por ocasião das cheias, a qual confere um substancial poder tampão (Abreu et al., 2007). Por sua vez, a acidez potencial $(\mathrm{H}+\mathrm{Al})$ foi significativamente menor na FS,

Tabela 2. Atributos químicos dos solos das diferentes unidades da paisagem do Pantanal, na sub-região da Nhecolândia, no Mato Grosso do $\mathrm{Sul}^{(1)}$.

\begin{tabular}{|c|c|c|c|c|c|c|c|c|c|c|c|c|}
\hline $\begin{array}{l}\text { Unidades da } \\
\text { paisagem }^{(2)}\end{array}$ & $\mathrm{pH}\left(\mathrm{H}_{2} \mathrm{O}\right)$ & \multicolumn{4}{|c|}{ - $\left(\mathrm{cmol}_{\mathrm{c}} \mathrm{dm}^{-3}\right)$---------- } & $\begin{array}{c}\mathrm{K}^{+} \\
\left(\mathrm{mg} \mathrm{dm}^{-3}\right)\end{array}$ & \multicolumn{2}{|c|}{$\begin{array}{c}\text { SB } \quad \mathrm{CTC}_{\mathrm{t}} \\
---\left(\mathrm{cmol}_{\mathrm{c}} \mathrm{dm}^{-3}\right)----\end{array}$} & $\begin{array}{c}\mathrm{V} \\
-----\end{array}$ & $m$ & $\begin{array}{c}\mathrm{P} \\
\left(\mathrm{mg} \mathrm{dm}^{-3}\right)\end{array}$ & $\begin{array}{c}\mathrm{MOS} \\
\left(\mathrm{g} \mathrm{kg}^{-1}\right)\end{array}$ \\
\hline \multicolumn{13}{|c|}{ Profundidade de $0,00-0,05 \mathrm{~m}$} \\
\hline FS & $6,6 \mathrm{a}$ & $1,1 \mathrm{~d}$ & $0,0 \mathrm{~b}$ & $3,2 \mathrm{a}$ & $0,8 \mathrm{a}$ & $115,0 \mathrm{a}$ & $4,3 \mathrm{a}$ & $4,3 \mathrm{a}$ & $79 \mathrm{a}$ & $0 \mathrm{~b}$ & $19,5 \mathrm{a}$ & $19,7 \mathrm{a}$ \\
\hline $\mathrm{CE}$ & $5,2 \mathrm{~b}$ & $1,8 \mathrm{~b}$ & $0,3 \mathrm{a}$ & $0,5 b c$ & $0,1 \mathrm{~b}$ & $31,0 \mathrm{c}$ & $0,7 \mathrm{~b}$ & $1,1 \mathrm{~b}$ & $28 b$ & $34 \mathrm{a}$ & $11,2 \mathrm{~b}$ & $10,7 \mathrm{~b}$ \\
\hline $\mathrm{CC}$ & $5,1 \mathrm{~b}$ & $1,4 \mathrm{c}$ & $0,3 \mathrm{a}$ & $0,3 \mathrm{c}$ & $0,2 \mathrm{~b}$ & $24,0 \mathrm{c}$ & $0,6 \mathrm{~b}$ & $0,9 \mathrm{~b}$ & $29 b$ & $36 \mathrm{a}$ & $5,2 \mathrm{bc}$ & $7,0 \mathrm{~b}$ \\
\hline CLE & $5,6 \mathrm{~b}$ & $1,6 \mathrm{bc}$ & $0,3 \mathrm{a}$ & $0,2 \mathrm{c}$ & $0,1 \mathrm{~b}$ & $91,7 \mathrm{ab}$ & $0,6 \mathrm{~b}$ & $0,9 \mathrm{~b}$ & $26 \mathrm{~b}$ & $34 \mathrm{a}$ & $5,4 \mathrm{bc}$ & $10,3 \mathrm{~b}$ \\
\hline CLA & $5,4 \mathrm{~b}$ & $1,7 \mathrm{~b}$ & $0,2 \mathrm{a}$ & $0,5 b c$ & $0,1 \mathrm{~b}$ & $24,0 \mathrm{c}$ & $0,6 \mathrm{~b}$ & $0,9 \mathrm{~b}$ & $27 \mathrm{~b}$ & $28 \mathrm{a}$ & $4,2 \mathrm{c}$ & $10,7 \mathrm{~b}$ \\
\hline BB & $5,0 \mathrm{~b}$ & $2,3 a$ & $0,2 \mathrm{a}$ & $0,7 \mathrm{~b}$ & $0,1 \mathrm{~b}$ & $13,3 \mathrm{c}$ & $0,8 \mathrm{~b}$ & $1,1 \mathrm{~b}$ & $27 \mathrm{~b}$ & $23 a b$ & $3,3 \mathrm{c}$ & $17,0 \mathrm{a}$ \\
\hline VB & $5,3 \mathrm{~b}$ & $1,9 \mathrm{~b}$ & $0,3 \mathrm{a}$ & $0,4 \mathrm{c}$ & $0,1 \mathrm{~b}$ & $45,3 \mathrm{bc}$ & $0,6 \mathrm{~b}$ & $0,9 \mathrm{~b}$ & $24 \mathrm{~b}$ & $32 \mathrm{a}$ & $2,3 \mathrm{c}$ & $11,3 \mathrm{~b}$ \\
\hline \multicolumn{13}{|c|}{ Profundidade de $0,05-0,10 \mathrm{~m}$} \\
\hline FS & $6,5 \mathrm{a}$ & $1,2 \mathrm{~d}$ & $0,0 \mathrm{c}$ & $2,3 \mathrm{a}$ & $0,6 \mathrm{a}$ & $86,3 \mathrm{a}$ & $3,1 \mathrm{a}$ & $3,1 \mathrm{a}$ & $72 \mathrm{a}$ & $0 \mathrm{~b}$ & $11,3 \mathrm{a}$ & $13,0 \mathrm{a}$ \\
\hline $\mathrm{CE}$ & $4,9 \mathrm{~cd}$ & $1,8 \mathrm{ab}$ & $0,5 \mathrm{a}$ & $0,2 \mathrm{~b}$ & $0,1 b$ & $20,0 \mathrm{~b}$ & $0,3 b$ & $0,8 \mathrm{~b}$ & $16 \mathrm{~b}$ & $57 \mathrm{a}$ & $14,4 \mathrm{a}$ & $7,7 \mathrm{bc}$ \\
\hline $\mathrm{CC}$ & $5,0 \mathrm{~cd}$ & $1,3 \mathrm{~cd}$ & $0,4 \mathrm{ab}$ & $0,1 \mathrm{~b}$ & $0,1 \mathrm{~b}$ & $9,3 b$ & $0,2 b$ & $0,6 \mathrm{~b}$ & $16 \mathrm{~b}$ & $61 \mathrm{a}$ & $2,6 b$ & $4,7 \mathrm{c}$ \\
\hline CLE & $5,7 \mathrm{~b}$ & $1,4 \mathrm{~cd}$ & $0,3 b$ & $0,1 \mathrm{~b}$ & $0,1 \mathrm{~b}$ & $31,2 \mathrm{~b}$ & $0,3 b$ & $0,6 \mathrm{~b}$ & $20 \mathrm{~b}$ & $43 a$ & $3,0 \mathrm{~b}$ & $7,7 \mathrm{bc}$ \\
\hline CLA & $5,3 \mathrm{bcd}$ & $1,4 \mathrm{~cd}$ & $0,3 b$ & $0,2 \mathrm{~b}$ & $0,1 \mathrm{~b}$ & $14,0 \mathrm{~b}$ & $0,3 b$ & $0,6 \mathrm{~b}$ & $19 b$ & $45 \mathrm{a}$ & $2,6 b$ & $7,7 \mathrm{bc}$ \\
\hline BB & $4,8 \mathrm{~d}$ & $2,0 \mathrm{a}$ & $0,4 \mathrm{ab}$ & $0,3 \mathrm{~b}$ & $0,1 \mathrm{~b}$ & $9,0 \mathrm{~b}$ & $0,4 \mathrm{~b}$ & $0,7 \mathrm{~b}$ & $16 \mathrm{~b}$ & $49 \mathrm{a}$ & $2,7 \mathrm{~b}$ & $11,3 \mathrm{ab}$ \\
\hline VB & $5,5 \mathrm{bc}$ & $1,6 \mathrm{bc}$ & $0,3 b$ & $0,2 \mathrm{~b}$ & $0,1 \mathrm{~b}$ & 37,3ab & $0,4 \mathrm{~b}$ & $0,7 \mathrm{~b}$ & $20 \mathrm{~b}$ & $40 \mathrm{a}$ & $2,2 b$ & $8,0 \mathrm{bc}$ \\
\hline \multicolumn{13}{|c|}{ Profundidade de $0,10-0,15 \mathrm{~m}$} \\
\hline FS & $6,4 \mathrm{a}$ & $1,2 \mathrm{c}$ & $0,0 \mathrm{c}$ & $1,9 \mathrm{a}$ & $0,5 \mathrm{a}$ & $85,0 \mathrm{a}$ & $2,5 \mathrm{a}$ & $2,5 \mathrm{a}$ & $68 \mathrm{a}$ & $0 \mathrm{~b}$ & $8,4 \mathrm{~b}$ & $9,7 \mathrm{a}$ \\
\hline $\mathrm{CE}$ & $4,8 \mathrm{c}$ & $1,8 \mathrm{~b}$ & $0,5 \mathrm{a}$ & $0,2 \mathrm{~b}$ & $0,1 \mathrm{~b}$ & $16,0 \mathrm{~b}$ & $0,3 b$ & $0,8 \mathrm{~b}$ & $14 \mathrm{~b}$ & $62 \mathrm{a}$ & $20,1 \mathrm{a}$ & $5,7 \mathrm{ab}$ \\
\hline $\mathrm{CC}$ & $5,0 \mathrm{bc}$ & $1,3 \mathrm{c}$ & $0,4 a b$ & $0,1 \mathrm{~b}$ & $0,1 \mathrm{~b}$ & $6,0 \mathrm{~b}$ & $0,2 b$ & $0,6 \mathrm{~b}$ & $14 \mathrm{~b}$ & $65 a$ & $1,9 \mathrm{c}$ & $4,0 \mathrm{~b}$ \\
\hline CLE & $5,7 \mathrm{~b}$ & $1,3 \mathrm{c}$ & $0,3 b$ & $0,1 \mathrm{~b}$ & $0,1 \mathrm{~b}$ & $12,0 \mathrm{~b}$ & $0,2 b$ & $0,5 b$ & $15 \mathrm{~b}$ & $57 \mathrm{a}$ & $2,3 \mathrm{c}$ & $6,0 \mathrm{ab}$ \\
\hline CLA & $5,3 \mathrm{bc}$ & $1,3 \mathrm{c}$ & $0,3 b$ & $0,2 \mathrm{~b}$ & $0,1 \mathrm{~b}$ & $12,0 \mathrm{~b}$ & $0,3 \mathrm{~b}$ & $0,6 \mathrm{~b}$ & $18 \mathrm{~b}$ & $51 \mathrm{a}$ & $2,8 \mathrm{bc}$ & $5,7 \mathrm{ab}$ \\
\hline BB & $4,7 \mathrm{c}$ & $2,2 \mathrm{a}$ & $0,5 \mathrm{a}$ & $0,2 \mathrm{~b}$ & $0,1 \mathrm{~b}$ & $9,7 b$ & $0,3 \mathrm{~b}$ & $0,8 \mathrm{~b}$ & $13 b$ & $59 \mathrm{a}$ & $3,6 \mathrm{bc}$ & $9,3 \mathrm{a}$ \\
\hline VB & $5,6 \mathrm{~b}$ & $1,4 \mathrm{c}$ & $0,3 \mathrm{~b}$ & $0,1 \mathrm{~b}$ & $0,1 \mathrm{~b}$ & $36,7 \mathrm{ab}$ & $0,3 \mathrm{~b}$ & $0,6 \mathrm{~b}$ & $19 \mathrm{~b}$ & $45 \mathrm{a}$ & $1,8 \mathrm{c}$ & $6,0 \mathrm{ab}$ \\
\hline \multicolumn{13}{|c|}{ Profundidade de $0,15-0,20 \mathrm{~m}$} \\
\hline FS & $6,2 a$ & $1,1 \mathrm{~b}$ & $0,0 \mathrm{~d}$ & $1,5 \mathrm{a}$ & $0,5 \mathrm{a}$ & $71,3 \mathrm{a}$ & $2,1 \mathrm{a}$ & $2,1 \mathrm{a}$ & $65 a$ & $0 \mathrm{~b}$ & $8,0 \mathrm{~b}$ & $8,3 \mathrm{ab}$ \\
\hline $\mathrm{CE}$ & $4,9 \mathrm{c}$ & $1,8 \mathrm{~b}$ & $0,5 \mathrm{a}$ & $0,1 \mathrm{~b}$ & $0,1 \mathrm{~b}$ & $15,7 \mathrm{~b}$ & $0,3 b$ & $0,8 b$ & $14 \mathrm{~b}$ & $66 a$ & $19,0 \mathrm{a}$ & $5,0 a b c$ \\
\hline $\mathrm{CC}$ & $5,0 \mathrm{bc}$ & $1,2 b$ & $0,4 a b$ & $0,1 \mathrm{~b}$ & $0,1 \mathrm{~b}$ & $5,0 \mathrm{~b}$ & $0,2 b$ & $0,6 \mathrm{~b}$ & $14 \mathrm{~b}$ & $67 \mathrm{a}$ & $1,8 \mathrm{c}$ & $3,3 \mathrm{c}$ \\
\hline CLE & $5,3 \mathrm{bc}$ & $1,3 b$ & $0,3 b c$ & $0,1 \mathrm{~b}$ & $0,1 \mathrm{~b}$ & $11,0 \mathrm{~b}$ & $0,2 \mathrm{~b}$ & $0,5 \mathrm{~b}$ & $15 b$ & $57 \mathrm{a}$ & $2,2 \mathrm{bc}$ & $5,3 a b c$ \\
\hline CLA & $5,2 \mathrm{bc}$ & $1,3 b$ & $0,3 b c$ & $0,1 \mathrm{~b}$ & $0,1 b$ & $10,0 \mathrm{~b}$ & $0,2 \mathrm{~b}$ & $0,5 \mathrm{~b}$ & $17 b$ & $51 \mathrm{ab}$ & $2,7 \mathrm{bc}$ & $4,3 b c$ \\
\hline BB & $4,8 \mathrm{c}$ & $2,0 \mathrm{a}$ & $0,5 \mathrm{a}$ & $0,2 \mathrm{~b}$ & $0,1 \mathrm{~b}$ & $11,0 \mathrm{~b}$ & $0,3 b$ & $0,8 b$ & $14 \mathrm{~b}$ & $58 \mathrm{a}$ & $3,8 \mathrm{bc}$ & $9,0 \mathrm{a}$ \\
\hline VB & $5,6 \mathrm{~b}$ & $1,1 \mathrm{~b}$ & $0,2 \mathrm{c}$ & $0,1 \mathrm{~b}$ & $0,1 \mathrm{~b}$ & $42,0 \mathrm{ab}$ & $0,3 b$ & $0,5 \mathrm{~b}$ & $23 b$ & $31 \mathrm{~b}$ & $1,5 \mathrm{c}$ & $4,0 \mathrm{bc}$ \\
\hline
\end{tabular}

${ }^{(1)}$ Médias seguidas de letras iguais, nas colunas e na mesma profundidade, não diferem pelo teste de Tukey, a $5 \%$ de probabilidade. (2)FS, floresta semidecídua; CE, cerradão; CC, cerrado/campo cerrado; CLE, campo limpo com predominância de Elionurus muticus; CLA, campo limpo com predominância de Axonopus purpusii e Andropogon spp.; BB, bordas de baías; e VB, vazantes/baixadas. SB, soma de bases trocáveis; $\mathrm{CTC}_{\mathrm{t}}$, capacidade de troca de cátions efetiva; $\mathrm{V}$, saturação por bases; $\mathrm{m}$, saturação por $\mathrm{Al}^{3+}$; $\mathrm{P}$, fósforo disponível; e MOS, matéria orgânica do solo. 
representada apenas por íons $\mathrm{H}^{+}$e pela ausência de $\mathrm{Al}^{3+}$, o que pode ser consequência do maior efeito da complexação do $\mathrm{Al}^{3+}$ por compostos orgânicos (Campos et al., 2011), presentes em maior grau nos solos dessa unidade da paisagem.

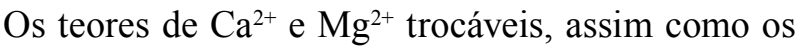
valores de $\mathrm{SB}, \mathrm{CTC}_{\mathrm{t}}$ e $\mathrm{V}$, foram significativamente maiores na FS, e não diferiram nos solos das demais unidades da paisagem, independentemente da profundidade avaliada (Tabela 2). Também para o $\mathrm{P}$ disponível, os maiores valores foram observados na FS, o que corrobora os resultados de Abreu et al. (2007), que destacaram que a dinâmica deste nutriente está estreitamente ligada ao aumento do $\mathrm{pH}$, como de fato ocorreu no solo dessa unidade da paisagem. Já os teores de $\mathrm{K}^{+}$, apesar de superiores na FS, também diferiram em profundidade nas demais unidades da paisagem, com destaque para valores mais altos no CLE e nas VB. O Ca ${ }^{2+}$ e o $\mathrm{Mg}^{2+}$ trocáveis fixaram-se nos horizontes superficiais, especialmente em razão da ciclagem vegetal, enquanto a distribuição do $\mathrm{K}^{+}$no perfil depende fortemente da mineralogia da fração argila, uma vez que o nutriente é fixado na estrutura das ilitas, presentes nesses solos (Coringa et al., 2014). Nos solos adjacentes às lagoas salinas, foram encontrados argilominerais formados in situ, principalmente por meio da precipitação de íons, como $\mathrm{Mg}^{2+}, \mathrm{Si}^{4+}$ e $\mathrm{K}^{+}$, decorrente da concentração evaporatória das águas superficiais e subsuperficiais (Furquim et al., 2008, 2010).

Apesar da generalizada baixa fertilidade natural do solo nas diferentes unidades da paisagem, constatouse que ela variou significativamente, com tendência à melhor qualidade nas coberturas vegetais livres de inundação, representadas pela FS. Segundo GamaRodrigues et al. (2008), mesmo em condição de baixa fertilidade natural do solo, os atributos químicos permitem separar diferentes coberturas vegetais, no Estado do Rio de Janeiro, em dois grupos, e o $\mathrm{pH}$, seguido da $\mathrm{SB}$ e do $\mathrm{Al}^{3+}$, são os atributos que mais contribuíram para essa distinção. No entanto, Carvalho et al. (2005), apesar de observarem diferença significativa na fertilidade natural do solo em gradiente topográfico de mata ripária, identificaram o regime de umidade como principal fator ambiental associado à distribuição das espécies arbóreo-arbustivas na área.

Em relação à MOS, embora apresente conteúdos significativamente superiores na FS e nas BB, estes mostraram-se em níveis relativamente baixos (menores que $20 \mathrm{~g} \mathrm{~kg}^{-1}$ ), enquanto, nas demais unidades da paisagem, a partir de $0,10 \mathrm{~m}$ de profundidade, eles foram classificados como muito baixos (menores que $6 \mathrm{~g} \mathrm{~kg}^{-1}$ ) (Tabela 2). Sob vegetação natural, o conteúdo de MOS é estável (Bortolon et al., 2009); portanto, como as unidades da paisagem analisadas são sistemas naturais, sem nenhum tipo de insumo aplicado ao solo, os resultados indicam reduzido aporte de material orgânico. Contudo, tendo-se em mente a textura arenosa e a baixa fertilidade natural desses solos, esses resultados já eram esperados, pois os campos naturais do Pantanal submetidos ao pastejo contínuo apresentam baixa capacidade de formação de fitomassa morta e caracterizam-se como ecossistemas marcados apenas pela extração de biomassa, basicamente sem nenhuma reposição ao solo, além dos excrementos de animais (Cardoso et al., 2011).

Os teores de MOS mais elevados nas BB e, sobretudo, na FS, podem ser atribuídos, no primeiro caso, à deposição de resíduos orgânicos pelo ciclo anual de cheia, especialmente de macrófitas aquáticas; à qualidade da MOS, composta possivelmente por formas mais recalcitrantes e mais estáveis (Lima et al., 2008), dominantes em termos quantitativos; ou, ainda, a menores taxas de decomposição, na ausência temporária de $\mathrm{O}_{2}$ (Nascimento et al., 2009). No caso da FS, os teores mais elevados certamente estiveram associados ao aporte de material orgânico na serapilheira, proveniente da FS. Resultados semelhantes foram relatados por Cardoso et al. (2011) e também associados ao maior aporte, de forma contínua e com conteúdo variado, de material orgânico proveniente de vegetação com maior diversidade de espécies.

Os dendrogramas obtidos, independentemente do método de ligação utilizado, mostraram a existência de um grupo diferenciado dos demais (Figura 2 A), representado pela FS, em todas as profundidades avaliadas. Os dendrogramas também evidenciaram a existência de dois outros grupos, um representado pelo $\mathrm{CE}$ e outro pelas unidades da paisagem $\mathrm{CC}$, CLE, CLA, BB e VB. Neste terceiro grupo, apenas as amostras da unidade da paisagem BB puderem ser claramente diferenciadas. $\mathrm{O}$ dendrograma obtido por UPGMA apresentou índice de correlação cofenética de 0,9 , que evidencia excelente representação gráfica 

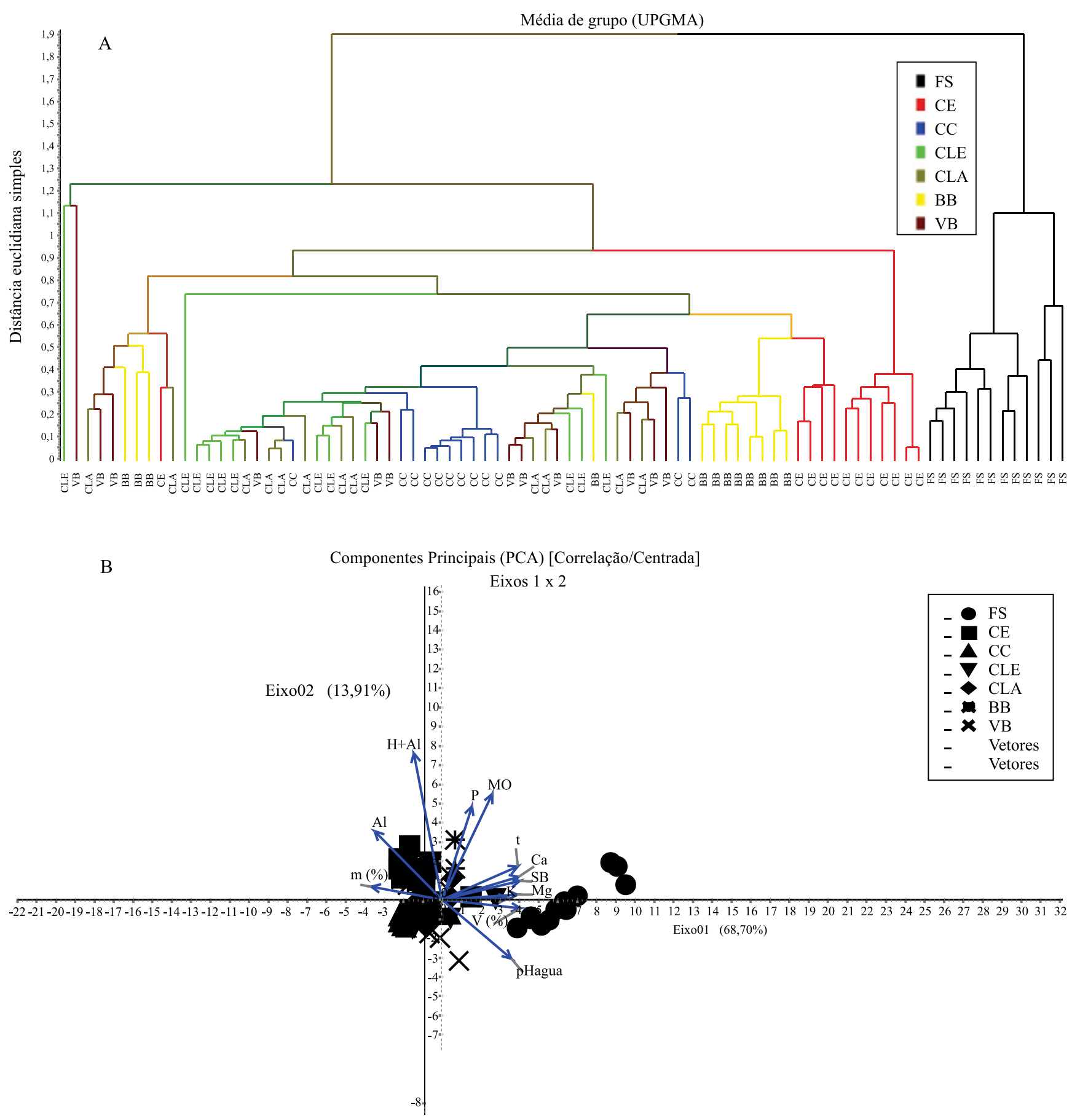

Figura 2. Análise multivariada dos atributos químicos dos solos das diferentes unidades da paisagem do Pantanal, na subregião da Nhecolândia, no Mato Grosso do Sul, obtido por meio de: A, dendograma obtido por método de ligação média entre grupos (UPGMA) e índice de distância euclidiana simples; e B, análise de componentes principais (PCA). FS, floresta semidecídua; CE, cerradão; CC, cerrado/campo cerrado; CLE, campo limpo com predominância de Elionurus muticus; CLA, campo limpo com predominância de Axonopus purpusii e Andropogon spp.; BB, bordas de baías; e VB, vazantes/ baixadas. $\mathrm{MO}$, matéria orgânica; $\mathrm{CTC}_{\mathrm{t}}$, capacidade de troca de cátions efetiva; $\mathrm{SB}$, soma de bases trocáveis; $\mathrm{V}$, saturação por bases; e m, saturação por $\mathrm{Al}^{3+}$. 
da matriz de distâncias euclidianas original, razão pela qual apenas ele foi apresentado (Figura 2).

O eixo 1 da PCA, obtida pela matriz de correlação centrada, explicou $64,3 \%$ da variância; o eixo 2 , $16,1 \%$; e o eixo 3, 9,6\% (Figura 2 B). Desse modo, $80,4 \%$ do total da variância puderam ser explicados pelos dois primeiros eixos da análise, e apenas $10 \%$ dessa variância se deveram a fatores não avaliados. A PCA confirmou os resultados das análises de agrupamento. $\mathrm{O}$ gráfico mostrou a existência de uma diferenciação clara da unidade da paisagem FS das demais, no eixo 1 , que se deveu à melhor qualidade dos atributos químicos do solo, representada por: maiores concentrações de $\mathrm{Mg}^{2+}, \mathrm{Ca}^{2+}, \mathrm{K}^{+}$e MOS; maiores valores de $\mathrm{V}$ e $\mathrm{pH}$; e menores valores de acidez potencial, $\mathrm{m} \%$ e $\mathrm{Al}^{3+}$ (Figura $2 \mathrm{~B}$ e Tabela 2). Já a unidade da paisagem $\mathrm{CE}$ pôde ser diferenciada das unidades savânicas e campestres (CC, CLE, CLA, BB e VB) no eixo 2 da análise, por ter apresentado maiores valores de $\mathrm{P}$ disponível. A unidade BB mostrou-se diferente das demais, incluindo as campestres, por apresentar valores de acidez potencial e de MOS mais elevados. A unidade BB representa áreas mais úmidas que, em geral, apresentam anaerobiose durante o período da cheia, o que favorece o acúmulo de matéria orgânica (Alewell et al., 2008; Mello et al., 2015). As unidades CC, CLE, CLA e VB, porém, não se diferenciaram entre si quanto aos atributos químicos do solo. Assim, não houve correspondência de sua diferenciação com os atributos mensurados. Embora as unidades CLA e VB sofram inundação sazonal, estas áreas podem apresentar o efeito Birch, citado por Mello et al. (2015), fenômeno que ocorre em áreas que sofrem ciclos consecutivos de cheia e seca, com consequente aumento da mineralização da MOS. De maneira geral, as unidades da paisagem puderam ser distinguidas de acordo com o gradiente e a influência da inundação em áreas florestadas, em formações savânicas/campos altos e sazonais, e em áreas úmidas.

\section{Conclusões}

1. Os atributos físico-químicos dos solos, nas diferentes unidades da paisagem avaliadas na sub-região da Nhecolândia, no Pantanal do Mato Grosso do Sul, evidenciam condições naturais ácidas, textura arenosa, baixo teor de matéria orgânica, e baixa capacidade de reter cátions e ânions.
2. A principal diferenciação entre os solos é representada pelas fertilidade natural mais elevada, na floresta semidecídua (FS), em especial na camada superficial e abaixo de $2 \mathrm{~m}$ de profundidade, com destaque para a concentração de $\mathrm{Na}^{+}$relativamente alta e para os teores mais elevados de $\mathrm{P}$ disponível, aliada ao gradiente topográfico.

3. As unidades FS e bordas de baías (BB) acumulam os maiores teores de matéria orgânica do solo, condicionados pelo maior aporte de substrato orgânico na serapilheira e pelo ciclo de cheia, respectivamente.

4. As unidades da paisagem são discriminadas em três diferentes grupos, em função da qualidade dos atributos químicos do solo: $\mathrm{FS}$; cerradão (CE); e cerrado/campo cerrado (CC), campo limpo com Elionurus muticus (CLE), campo limpo com Axonopus purpusii e Andropogon spp. (CLA), vazantes/baixadas (VB) e bordas de baías (BB).

5. As diferenças fitofisionômicas entre unidades savânicas e campestres (CC, CLE, CLA, VB e BB) relacionam-se à frequência e ao grau de inundação sazonal.

\section{Referências}

ABREU, E.M.A. de; FERNANDES, A.R.; RUIVO, M. de L.P. Variação temporal e vertical de atributos químicos de um Gleissolo do rio Guamá cultivado com Canaranas. Revista Brasileira de Ciência do Solo, v.31, p.277-285, 2007. DOI: 10.1590/S010006832007000200010.

ALEWELL, C.; PAUL, S.; LISCHEID, G.; STORCK, F.R. Coregulation of redox processes in freshwater wetlands as a function of organic matter availability? Science of the Total Environment, v.404, p.335-342, 2008. DOI: 10.1016/j.scitotenv.2007.11.001.

ALVAREZ V., V.H.; NOVAIS, R.F.; BARROS, N.F. de; CANTARUTTI, R.B.; LOPES, A.S. Interpretação dos resultados das análises de solos. In: RIBEIRO, A.C.; GUIMARAES, P.T.G.; ALVAREZ V., V.H., (Ed.). Recomendações para o uso de corretivos e fertilizantes em Minas Gerais: $5^{\text {a }}$ aproximação. Viçosa: Comissão de Fertilidade do Solo do Estado de Minas Gerais, 1999. p.25-32.

BARBIERO, L.; REZENDE FILHO, A.; FURQUIM, S.A.C.; FURIAN, S.; SAKAMOTO, A.Y.; VALLES, V.; GRAHAM, R.C.; FORT, M.; FERREIRA, R.P.D.; QUEIROZ NETO, J.P. Soil morphological control on saline and freshwater lake hydrogeochemistry in the Pantanal of Nhecolândia, Brazil. Geoderma, v.148, p.91-106, 2008. DOI: 10.1016/j. geoderma.2008.09.010.

BORTOLON, E.S.O.; MIELNICZUK, J.; TORNQUIST, C.G.; LOPES, F.; FERNANDES, F.F. Simulação da dinâmica do carbono e nitrogênio em um Argissolo do Rio Grande do Sul usando modelo Century. Revista Brasileira de Ciência do Solo, v.33, p.1635-1646, 2009. DOI: 10.1590/S0100-06832009000600012. 
CAMPOS, L.P.; LEITE, L.F.C.; MACIEL, G.A.; IWATA, B. de F.; NÓBREGA, J.C.A. Atributos químicos de um Latossolo Amarelo sob diferentes sistemas de manejo. Pesquisa Agropecuária Brasileira, v.46, p.1681-1689, 2011. DOI: 10.1590/S0100204X2011001200014.

CARDOSO, E.L.; SILVA, M.L.N.; CURI, N.; FERREIRA, M.M.; FREITAS, D.A.F. de. Qualidade química e física do solo sob vegetação arbórea nativa e pastagens no Pantanal Sul-MatoGrossense. Revista Brasileira de Ciência do Solo, v.35, p.613-622, 2011. DOI: 10.1590/S0100-06832011000200030.

CARVALHO FILHO, A. de; CARDOSO, E.L.; NAIME, U.J.; MOTTA, P.E.F. da; OLIVEIRA, H. de; BRANCO, O.D.; SANTOS, R.D. dos. Solos como fator de diferenciação fitofisionômica na subregião da Nhecolândia - Pantanal Mato-Grossense. In: SIMPÓSIO SOBRE RECURSOS NATURAIS E SÓCIO-ECONÔMICOS DO PANTANAL, 3., 2000, Corumbá. Anais. Corumbá: Embrapa Pantanal, 2000. p.59-60.

CARVALHO, D.A.; OLIVEIRA FILHO, A.T.; VILELA, E.A.; CURI, N.; BERG, E. van den; FONTES, M.A.L.; BOTEZELLI, L. Distribuição de espécies arbóreo-arbustivas ao longo de um gradiente de solos e topografia em um trecho de floresta ripária do Rio São Francisco em Três Marias, MG, Brasil. Revista Brasileira de Botânica, v.28, p.329-345, 2005. DOI: 10.1590/S010084042005000200013 .

CLAESSEN, M.E.C. (Org.). Manual de métodos de análise de solo. 2.ed. rev. e atual. Rio de Janeiro: Embrapa-CNPS, 1997. 212p. (EMBRAPA-CNPS. Documentos, 1).

CORINGA, E. de A.O.; COUTO, E.G.; TORRADO, P.V. Geoquímica de solos do Pantanal Norte, Mato Grosso. Revista Brasileira de Botânica, v.38, p.1784-1793, 2014. DOI: 10.1590/ s0100-06832014000600013.

COUTINHO, L.M. O conceito de bioma. Acta Botanica Brasilica, v.20, p.13-23, 2006. DOI: 10.1590/S0102-33062006000100002.

CUNHA, N.G. da. Considerações sobre os solos da sub-região da Nhecolândia, Pantanal Mato-Grossense. Corumbá: EMBRAPAUEPAE de Corumbá, 1980. 45p. (EMBRAPA-UEPAE de Corumbá. Circular técnica, 1).

FURQUIM, S.A.C.; GRAHAM, R.C.; BARBIERO, L.; QUEIROZ NETO, J.P.; VALLÈS, V. Mineralogy and genesis of smectites in an alkaline-saline environment of Pantanal wetland, Brazil. Clays and Clay Minerals, v.56, p.579-595, 2008. DOI: 10.1346/ CCMN.2008.0560511.

FURQUIM, S.A.C.; GRAHAM, R.C.; BARBIERO, L.; QUEIROZ NETO, J.P.; VIDAL-TORRADO, P. Soil mineral genesis and distribution in a saline lake landscape of the Pantanal wetland, Brazil. Geoderma, v.154, p.518-528, 2010. DOI: 10.1016/j. geoderma.2009.03.014.

GAMA-RODRIGUES, E.F. da; GAMA-RODRIGUES, A.C. da; PAULINO, G.M.;FRANCO, A.A. Atributos químicos emicrobianos de solos sob diferentes coberturas vegetais no Norte do estado do Rio de Janeiro. Revista Brasileira de Ciência do Solo, v.32, p.1521-1530, 2008. DOI: 10.1590/S0100-06832008000400016.
JACKSON, J.E. A user's guide to principal components. New York: J. Wiley \& Sons, 1991. DOI: 10.1002/0471725331.

LIMA, A.M.N.; SILVA, I.R. da; NEVES, J.C.L.; NOVAIS, R.F. de, BARROS, N.F. de; MENDONÇA, E. de S.; DEMOLINARI, M. de S.M.; LEITE, F.P. Frações da matéria orgânica do solo após três décadas de cultivo de eucalipto no Vale do Rio Doce-MG. Revista Brasileira de Ciência do Solo, v.32, p.1053-1063, 2008. DOI: 10.1590/S0100-06832008000300014.

MELLO, J.M.; COUTO, E.G.; AMORIM, R.S.S.; CHIG, L.A.; JOHNSON, M.S.; LOBO, F.A. Dinâmica dos atributos físicoquímicos e variação sazonal dos estoques de carbono no solo em diferentes fitofisionomias do Pantanal Norte Mato-Grossense. Revista Árvore, v.39, p.325-336, 2015. DOI: 10.1590/010067622015000200012 .

NASCIMENTO, P.C. do; BAYER, C.; SILVA NETTO, L. de F. da; VIAN, A.C.; VIEIRO, F.; MACEDO, V.R.M.; MARCOLIN, E. Sistemas de manejo e a matéria orgânica de solo de várzea com cultivo de arroz. Revista Brasileira de Ciência do Solo, v.33, p.1821-1827, 2009. DOI: 10.1590/S0100-06832009000600030.

SALIS, S.M.; ASSIS, M.A.; CRISPIM, S.M.A.; CASAGRANDE, J.C. Distribuição e abundância de espécies arbóreas em cerradões no Pantanal, Estado do Mato Grosso do Sul, Brasil. Revista Brasileira de Botânica, v.29, p.339-352, 2006. DOI: 10.1590/ S0100-84042006000300002.

SANTOS, H.G. dos; JACOMINE, P.K.T.; ANJOS, L.H.C. dos; OLIVEIRA, V.A. de; LUMBRERAS, J.F.; COELHO, M.R.; ALMEIDA, J.A. de; CUNHA, T.J.F.; OLIVEIRA, J.B. de. Sistema brasileiro de classificação de solos. 3.ed. rev. e ampl. Brasília: Embrapa 2013. 353p.

SANTOS, R.D. dos; LEMOS, R.C. de; SANTOS, H.G. dos; KER, J.C.; ANJOS, L.H.C. dos. Manual de descrição e coleta de solos no campo. 5.ed. rev. e ampl. Viçosa: Sociedade Brasileira de Ciência do Solo; Rio de Janeiro: Embrapa-CNPS, 2005. 92p.

SANTOS, S.A.; COSTA, C.; SOUZA, G. da S. e; POTT, A.; ALVAREZ, J.M.; MACHADO, S.R. Composição botânica da dieta de bovinos em pastagem nativa na sub-região da Nhecolândia, Pantanal. Revista Brasileira de Zootecnia, v.31, p.1648-1662, 2002. DOI: 10.1590/S1516-35982002000700007.

SILVA, J. dos S.V. da; ABDON, M. de M. Delimitação do Pantanal brasileiro e suas sub-regiões. Pesquisa Agropecuária Brasileira, v.33, p.1703-1711, 1998. Número especial.

SKORUPA, A.L.A.; GUILHERME, L.R.G.; CURI, N.; SILVA, C.P. de C.; SCOLFORO, J.R.S.; MARQUES, J.J.G. de S. e M. Propriedades de solos sob vegetação nativa em Minas Gerais: distribuição por fitofisionomia, hidrografia e variabilidade espacial. Revista Brasileira de Ciência do Solo, v.36, p.11-22, 2012. DOI: 10.1590/S0100-06832012000100002.

SOMMER, M. Influence of soil pattern on matter transport in and from terrestrial biogeosystems - a new concept for landscape pedology. Geoderma, v.133, p.107-123, 2006. DOI: 10.1016/j. geoderma.2006.03.040.

$\overline{\text { Recebido em } 31 \text { de agosto de } 2015 \text { e aprovado em } 11 \text { de julho de } 2016}$

Pesq. agropec. bras., Brasília, v.51, n.9, p.1231-1240, set. 2016

DOI: 10.1590/S0100-204X2016000900023 